\title{
European Union Standards for Tuberculosis Care
}

\author{
G.B. Migliori, J.P. Zellweger, I. Abubakar, E. Ibraim, J.A. Caminero, G. De Vries, \\ L. D’Ambrosio, R. Centis, G. Sotgiu, O. Menegale, K. Kliiman, T. Aksamit, D.M. Cirillo, \\ M. Danilovits, M. Dara, K. Dheda, A.T. Dinh-Xuan, H. Kluge, C. Lange, V. Leimane, \\ R. Loddenkemper, L.P. Nicod, M.C. Raviglione, A. Spanevello, V.Ø. Thomsen, M. Villar, \\ M. Wanlin, J.A. Wedzicha, A. Zumla, F. Blasi, E. Huitric, A. Sandgren and D. Manissero
}

ABSTRACT: The European Centre for Disease Prevention and Control (ECDC) and the European Respiratory Society (ERS) jointly developed European Union Standards for Tuberculosis Care (ESTC) aimed at providing European Union (EU)-tailored standards for the diagnosis, treatment and prevention of tuberculosis (TB).

The International Standards for TB Care (ISTC) were developed in the global context and are not always adapted to the EU setting and practices. The majority of EU countries have the resources and capacity to implement higher standards to further secure quality TB diagnosis, treatment and prevention. On this basis, the ESTC were developed as standards specifically tailored to the EU setting.

A panel of 30 international experts, led by a writing group and the ERS and ECDC, identified and developed the 21 ESTC in the areas of diagnosis, treatment, HIV and comorbid conditions, and public health and prevention. The ISTCs formed the basis for the 21 standards, upon which additional EU adaptations and supplements were developed.

These patient-centred standards are targeted to clinicians and public health workers, providing an easy-to-use resource, guiding through all required activities to ensure optimal diagnosis, treatment and prevention of TB. These will support EU health programmes to identify and develop optimal procedures for TB care, control and elimination.

KEYWORDS: European Respiratory Society, European Union Standards, guidelines, MDR-TB, TB

W ith more than 80,000 tuberculosis (TB) cases notified in the European Union and European Economic Area (EU/EEA) Member States in 2009 (EU/EEA countries comprise Austria, Belgium, Bulgaria, Cyprus, Czech Republic, Denmark, Estonia, Finland, France, Germany, Greece, Hungary, Iceland, Republic of Ireland, Italy, Latvia, Liechtenstein, Lithuania, Luxembourg, Malta, The Netherlands, Norway, Poland, Portugal, Romania, Slovakia, Slovenia, Spain, Sweden, UK; while Switzerland is not in the EEA, Swiss nationals have the same rights as EEA nationals), TB continues to be a priority public health challenge in this setting. Although several EU/EEA countries are progressing towards sustained low levels of TB incidence, the contrasts in
TB disease burden remain great within the subregion [1]. Drug-resistant TB and multidrug-resistant TB (MDR-TB) pose a specific public health threat in some countries. Furthermore, assessing the prevalence of HIV co-infection among TB cases is still compromised by suboptimal reporting in several countries [1-3].

While EU/EEA countries adopted the key principles of TB control and elimination through the European-specific, consensus-based documents born within the Wolfheze initiative [1], a uniform set of guidelines summarising the minimum standards clinicians and healthcare workers should look at to guide their practice, was still lacking. The recent finalisation of the International Standards for
AFFILIATIONS

For a full list of affiliations, please see the Acknowledgements section.

CORRESPONDENCE

G.B. Migliori

WHO Collaborating Centre for TB and

Lung Diseases,

Fondazione S. Maugeri

Care and Research Institute

Via Roncaccio 16

21049 Tradate

Italy

E-mail: giovannibattista.migliori@

fsm.it

Received:

Nov 212011

Accepted after revision:

Dec 232011

This article was modified in April 2016 to correct errors in the licence information. 
Tuberculosis Care (ISTC) [1-4], developed in 2009 by 50 experts from 15 countries to include the perspectives of several organisations and international societies (which endorsed them for universal use), offered the opportunity to tackle this gap in the EU.

The ISTC [1-4] prescribe a widely accepted level of TB care which guides all healthcare providers and clinicians, both public and private, in achieving optimal standards in managing patients who have, or are suspected of having, active TB. The 21 ISTCs [4] are organised into 4 sections: 1) standards for diagnosis, 2) standards for treatment, 3) standards for addressing HIV co-infection and other comorbid conditions and 4) standards for public health.

The standards, seen as "a living document that will be revised as technology, resources and circumstances change", are designed to complement existing national or international guidelines, and are consistent with World Health Organization (WHO) definitions and recommendations.

The specific characteristics of the EU/EEA Member States which justify the need to develop standards specifically tailored to the European context are [1, 2]:

1) The majority of EU/EEA countries have a low incidence of TB; however, a heterogeneous setting exists with a number of Member States having high and intermediate TB levels, with varying levels of MDR-TB and TB-HIV co-infection. Also a number of countries border non-EU countries with a higher TB and MDR-TB burden.

2) TB services are fully integrated and merged within the health system in the majority of EU/EEA countries. This presents peculiarities in allocating responsibilities for the optimal delivery of TB care.

3) The EU/EEA countries have a long-established tradition of TB control that has evolved over past decades. New tools and high standards of diagnosis and care are often implemented in EU/EEA countries.

4) The EU/EEA countries are committed to pursue elimination of $\mathrm{TB}$, sharing a common platform based on the Wolfheze documents; the Framework action plan to fight TB in the EU; Progressing towards TB elimination, and the European Centre of Disease Prevention and Control (ECDC)-driven surveillance system [5-14].

In spite of efforts to introduce ISTCs among EU/EEA healthcare providers (including clinicians), their implementation has been suboptimal. A tailored set of standards present the potential to improve acceptability among clinical networks.

\section{METHODS}

A collaborative process, jointly led by the ECDC and the European Respiratory Society (ERS), was initiated to adapt the ISTCs to the EU/EEA setting and to develop the EU Standards for Tuberculosis Care (ESTC). While ERS has taken the lead in developing the clinically-related standards, ECDC has done the same for the public health standards.

A panel of more than 30 experts was convened representing the ERS and other international societies (American Thoracic Society (ATS), ECDC, national TB programmes and civil society (affected communities)) and organisations (WHO,
International Union against Tuberculosis and Lung Diseases (the Union, Europe Region) and KNCV Tuberculosis Foundation). Within this group, a writing committee consisting of eight experts was identified to lead the consensus and writing process of the document.

A non-systematic review of the evidence relevant for the development of the ESTC was performed (including the peerreviewed manuscripts and systematic reviews/meta-analyses included in the ISTC document and those published after the second edition of the ISTC) to support the development of the document.

A Delphi process was performed to define the list of standards based on prior evidence [1, 2].

The panel of experts was asked to assign priorities to the first draft of 45 proposed standards and to complete the literature synopsis of the standards-supporting evidence with a score ranging from 5 (high relevance) to 1 (low relevance). Based on the results of the Delphi process, the draft concept of the standards and the literature synopsis were completed and circulated within the panel of experts for two rounds of review, comments and additions. Consensus was at this stage reached to formulate the standards around the existing four sections of the ISTCs (diagnosis, treatment, clinical management of TB/ HIV and comorbidities, and public health and prevention). The Delphi exercise was completed by $85 \%$ of the panel of experts, with the main score per area being high, ranging between 4.04 and 4.74. The literature synopsis was also scored and 21 references were added to the 97 initially proposed.

The ESTC have been specifically developed to complement the ISTCs and other existing guidelines, being based mainly, but not exclusively, on the existing gaps in case management that were identified in a recent European MDR-TB case management survey as well as the survey of TB surveillance systems in lowincidence countries, including countries of the EU/EEA [2].

In the process of developing the standards and reaching a consensus, the panel of experts further identified the need and added value of complementing the standards with supporting enablers. These are aimed as a resource for policy makers, clinicians, public health workers and stakeholders as a support to identify resources and suggestions on how best to adopt, adapt, introduce and implement the ESTC in their setting.

The concept of ESTC was first presented and discussed in a symposium at the 2010 ERS Congress in Barcelona. Three working meetings between ERS and ECDC experts and a vis-àvis meeting of the expert writing committee were organised in 2011, preceding circulation of the draft document to the entire expert panel. The draft ESTC document was presented and discussed further during a symposium at the September 2011 ERS conference in Amsterdam and finalised in October 2011 before going through an official clearance process of the ERS and the ECDC.

\section{HOW TO READ THE DOCUMENT}

Under four sections (diagnosis, treatment, HIV and comorbidities, and public health), 21 standards are defined, which correspond to those of the ISTC. Using the ISTC as a basis, the EU Standards for TB care were developed and are listed as either: 
1) "Valid": the corresponding ISTC has been maintained and is considered the EU standard. When an ISTC was maintained as "valid", none-to-minimal wording will have been adapted to update to the EU-definition; i.e. smear-positive cases are now defined as culture-positive in diagnosis standards. Otherwise the original wording from the ISTC has been maintained in order to ensure consistency and comparability between the two documents.

2) "Valid with an EU-adapted supplement": the corresponding ISTC has been maintained but with an additional EU component that covers the minimal requirement for standards in the EU; as defined by the expert groups and EU definitions.

3) "Replaces standard $x^{\prime \prime}$ : the corresponding ISTC has been modified extensively in order to fit in with EU definitions and requirements.

When necessary, notes are listed after each standard to further define and explain specific components that the expert group deemed important to clarify. Where a standard replaces an ISTC, the original wording of the ISTC is listed under the heading Replaced ISTC at the end of the document.

\section{STANDARDS FOR TB DIAGNOSIS}

Standard 1 (replaces ISTC $1^{*}$ )

All persons presenting with signs, symptoms, history or risk factors compatible with TB should be evaluated for pulmonary and/or extrapulmonary TB.

Notes

1) The most common symptoms of pulmonary $\mathrm{TB}$ are persistent cough with or without sputum production for more than 2-3 weeks [4], while haemoptysis (blood in mucus) is more rare. Respiratory symptoms can be accompanied by fever, night sweats and weight loss. These signs and symptoms are common in a wide range of respiratory conditions including acute respiratory infections and acute exacerbation of chronic obstructive pulmonary disease. It is also possible for patients to present with no signs and symptoms of disease [15]. For extrapulmonary TB, TB organ-specific signs and symptoms may occur.

2) It is important to investigate the history of the patient with regard to TB. For example, history of TB in the family context, history of previous contact with TB and previous TB diagnosis and/or treatment, and any condition attenuating the host immune system [4] are common risk factors for TB that should be considered as relevant to guide the correct diagnosis.

3) In the EU setting, TB is not the leading cause of persistent cough and, thus, cough is not the leading sign or symptom of $\mathrm{TB}$ disease. Based on its expert knowledge and experience of the EU setting and the current evidence [16] the expert group therefore adapted this first standard to better describe a patient who should be evaluated for TB disease in the EU-setting.

\section{Standard 2 (replaces ISTC $2^{\#}$ )}

All patients (adults, adolescents and children who are capable of producing sputum) suspected of having pulmonary TB should have at least two sputum specimens submitted for microscopic examination, culture and drug susceptibility testing (DST) in a quality-assured laboratory. When possible, at least one early morning specimen should be obtained. In countries, settings or populations in which MDR-TB is suspected in a patient, rapid testing for the identification of rifampicin- and isoniazid-resistance, using validated tools in a quality-assured laboratory, should be performed.

Notes

1) Based on the EU/EEA practice and definitions [13, 14, 17], quality-assured DST should be performed on all diagnosed TB patients to rule out drug resistance [5-14]. This should follow international standards and guidelines with regard to methods used and drug concentrations, for testing of first- and secondline drugs [8-10]. Samples sent for bacteriological examinations (sputum smear, culture, DST and new molecular methods) should be addressed to a mycobacteriology laboratory which implements optimal laboratory practices and quality-assured procedures according to European and international recommendations [8-10, 18-21].

2) There are two categories of risk factors for MDR-TB: patient risk factors and setting risk factors [22, 23], which need to be considered when assessing the risk for MDR-TB [24]. Previous contact with MDR-TB patients and previous history of TB treatment are two key patient risk factors for MDR-TB. Originating from settings with a high prevalence of MDR-TB (e.g. Eastern European countries) are further relevant risk factors for MDR-TB [18, 25-28].

3) Countries should transform the process of risk assessment into guidelines and adapt them to their national needs [4, 7].

4) Quality bacteriological diagnosis includes the WHO recommended rapid molecular assays, which should be performed as early as possible (ideally on the same day as diagnosis) within evidence-based diagnostic algorithms and guidelines. Currently available methods are the automated real-time nucleic acid amplification technology for rapid and simultaneous detection of Mycobacterium tuberculosis and rifampicin resistance, and the line probe assays for rapid $M$. tuberculosis detection and rifampicin resistance or rifampicin and/or isoniazid resistance testing $[19,29,30]$. These approaches allow immediate identification of $M$. tuberculosis and rifampicin resistance and/or MDR-TB (rifampicin resistance can be considered a proxy of MDR-TB) [19-21, 29, 30, 31]. All molecular diagnostic results must be confirmed further by culture-based DST. All identified MDR-TB cases should be managed by specialised centres directly upon diagnosis and a second independent molecular test with a different technology should be performed to confirm the rapid diagnosis [32-37].

5) The choice of molecular methods for the rapid identification of rifampicin resistance and isoniazid resistance should be that of WHO-endorsed rapid diagnostic assays [29].

6) Optimal, clear and direct communication between the laboratory experts and clinicians is essential to obtain the optimal link of diagnosis and choice of treatment. The clinician needs to be informed whether the laboratory performs DST for second-line drugs and on which drugs. Furthermore, they need to be informed on whether proficiency testing (external quality assurance) for all provided DST methods is implemented according to evidence-based guidelines. The clinician 
needs to ensure that the bacteriological sample undergoes first- and second-line DST according to evidence-based guidelines $[8-10,18,38]$.

7) Based on feasibility and cost-effectiveness analyses, the WHO and the ISTC recommend the collection of at least two sputum samples for diagnosis. Given that the collection of a third sample has been shown to increase the diagnostic yield by $2-3 \%$, EU/ EEA countries may decide to maintain the previous recommendation of collecting three sputum samples on the same day (not necessarily on consecutive days) $[4,39,40]$.

8) It is essential to obtain quality sputum samples in order to ensure quality and reliable bacteriological testing of the sample $[4,39]$. This includes ensuring appropriate collection, storage, transportation and processing of sputum samples, as well as obtaining at least one early morning sample from the patient.

9) All the procedures aimed at obtaining samples for culture and DST should be used whenever possible according to evidence-based guidelines (sputum induction, bronchoscopy and gastric lavage in children).

\section{Standard 3 (replaces ISTC $3^{4}$ )}

For all patients (adults, adolescents and children) suspected of having extrapulmonary $\mathrm{TB}$, appropriate specimens from the suspected sites of involvement should be obtained for microscopy, culture, DST and histopathological examination in a quality-assured laboratory. In countries, settings or populations in which MDR-TB is suspected in a patient, rapid testing for the identification of rifampicin and isoniazid resistance in a quality-assured laboratory could be performed.

\section{Notes}

1) This third standard has been accordingly updated to be in line with standards 1 and 2 with regard to essential, standard diagnosis.

2) It is essential to obtain bacteriological confirmation from extrapulmonary sites in order to confirm diagnosis and consequently provide optimal and effective treatment; this may include the more sensitive molecular test [4].

\section{Standard 4 (replaces ISTC $4^{+}$)}

All persons with chest radiographic findings suggestive of pulmonary TB should have sputum specimens submitted for microscopic examination, culture and DST in a quality-assured laboratory. In countries, settings or populations in which MDR-TB is suspected in a patient, rapid testing for the identification of rifampicin resistance and when possible isoniazid resistance in a quality-assured laboratory should be performed.

\section{Notes}

1) This standard has been updated to be consistent with the standards 1 and 2 with regard to essential, standard diagnosis [4].

\section{Standard 5 (ISTC 5 valid with an EU-adapted supplement)}

The diagnosis of culture-negative pulmonary TB should be based on the following criteria: all bacteriological tests are negative (including direct sputum smear examinations, cultures and rapid molecular testing); chest radiographic findings are compatible with TB; and there is a lack of response to a trial of broad spectrum antimicrobial agents (because the fluoroquinolones are active against $M$. tuberculosis complex and, thus, may cause transient improvement in persons with TB, they should be avoided). In persons who are seriously ill or have known or suspected HIV infection or have any immunecompromising conditions, the diagnostic evaluation should be expedited and, if clinical evidence strongly suggests $\mathrm{TB}$, a course of anti-TB treatment should be initiated.

\section{EU-adapted supplement}

As the EU has adopted the culture-based case definition, the main distinction of TB cases should be between culturepositive and -negative cases, and not sputum smear status [5, $8,11,41]$.

In order to ensure quality diagnosis of both pulmonary and extrapulmonary $\mathrm{TB}$, adequate samples for bacteriological examination should be obtained and processed using available diagnostic tools (e.g. induced sputum, bronchoscopy and bronchoalveolar lavage, and gastric washing) [4, 42-44], and complemented by imaging (radiology, computed tomography and magnetic resonance imaging) and other necessary examinations performed according to evidence-based guidelines [4, $32,35,45,46]$.

Culture, DST and rapid molecular testing should be performed on each sample from both pulmonary and extrapulmonary TB, including samples obtained through surgery or other invasive procedures which usually undergo histological examinations $[5,8,11,41]$.

Surgeons should be advised to save a biological specimen in normal saline for microbiological and molecular biological examinations and in formalin for histopathological examinations.

Notes

1) Other existing new diagnostic tools, e.g. additional nucleic acid amplification techniques and other new techniques in the development pipeline, after necessary validation has been achieved, should be used within evidence-based diagnostic algorithms and guidelines [19, 29, 46-54]. Before introducing any new tool or approach, the evidence has to be validated and have shown efficacy and patient value.

\section{Standard 6 (ISTC 6 valid until specific EU paediatric standards are available, with an EU-adapted supplement)}

In all children suspected of having intrathoracic (i.e. pulmonary, pleural, and mediastinal or hilar lymph node) TB, bacteriological confirmation should be sought through examination of appropriate biological samples (by expectoration or induced sputum, bronchial secretions, pleural fluid or gastric washings) for smear microscopy, culture and DST in a qualityassured laboratory. In the event of negative bacteriological results, a diagnosis of TB should be based on the presence of abnormalities consistent with TB on chest radiography or other imaging, a history of exposure to an infectious case, evidence of TB infection (positive tuberculin skin test (TST) and/or interferon $-\gamma$ release assay (IGRA)) and clinical findings 
suggestive of TB. For children suspected of having extrapulmonary $\mathrm{TB}$, appropriate specimens from the suspected sites of involvement should be obtained for microscopy and for culture and histopathological examination.

\section{EU-adapted supplement}

Once TB is confirmed in a child, as defined previously, standards $2-5$ should be followed for complete diagnosis of the case $[4,55]$.

Notes

1) With the exception of two clarifications (use of the term "appropriate biological samples"; and for smear microscopy, culture and DST in a quality-assured laboratory), this ESTC is the same as the ISTC 6 for paediatric diagnosis [4].

2) ISTC 6 is valid until specific EU paediatric standards are available.

3) Rapid DST testing could/should be considered in order to obtain a rapid, preliminary diagnosis. However, research is still ongoing to validate new molecular methods in this group.

\section{STANDARDS FOR TB TREATMENT}

\section{Standard 7 (ISTC 7 valid)}

Any practitioner treating a patient for TB is assuming an important public health responsibility to prevent ongoing transmission of the infection and the development of drug resistance. To fulfil this responsibility the practitioner must not only prescribe an appropriate regimen, but also utilise local public and/or community health services, agencies and resources when necessary, to perform contact investigation, to assess the adherence of the patient and to address poor adherence when it occurs.

Notes

1) The ISTC was maintained with the inclusion of naming the need to involve community health services so as to highlight the important role of civil society in patient treatment and adherence $[4,56,57]$.

\section{Standard 8 (ISTC 8 valid with an EU-adapted supplement)}

All patients (including those with HIV-infection) who have not been previously treated and without any risk factors for drug resistance should receive an internationally accepted first-line treatment regimen using drugs of known bioavailability. The initial phase should consist of two months of isoniazid, rifampicin, pyrazinamide and ethambutol. The continuation phase should consist of isoniazid and rifampicin given for four months (2HRZE/4HR). The doses of anti-TB drugs used should conform to international recommendations. Fixed dose combinations of two (isoniazid and rifampicin), three (isoniazid, rifampicin and pyrazinamide) and four (isoniazid, rifampicin, pyrazinamide and ethambutol) drugs are highly recommended.

\section{EU-adapted supplement}

The selection of standardised regimens for WHO category I and III patients (i.e. new pulmonary sputum smear-positive and new sputum smear-negative/extrapulmonary cases) should be performed according to international recommendations in centres having the necessary expertise, as defined at the national level and based on the number of cases managed every year and other relevant parameters [4, 32, 35, 40, 58, 59]. The clinician should ensure the correct drug regimen (including four active drugs for the intensive phase of treatment) at the correct dose for a sufficient duration. The daily dosage is recommended at least during the intensive phase of treatment.

Based on confirmed DST results, treatment must be adapted according to the drug-susceptibility pattern [4, 32, 34-37, 40, $58,59]$.

Ideally, MDR-TB should be excluded in all TB cases. Retreatment cases should be managed according to the individual risk of MDR-TB until multi-drug resistance is excluded [24, 33, 34, 36, 37, 60].

This standard has implications for the standards of diagnosis, treatment and infection control.

\section{Standard 9 (ISTC 9 valid)}

To assess and foster adherence, a patient-centred approach to administration of drug treatment, based on the patient's needs and mutual respect between the patient and the provider, should be developed for all patients.

Supervision and support should be individualised and should draw on the full range of recommended interventions and available support services, including patient counselling and education. A central element of the patient-centred strategy is the use of measures to assess and promote adherence to the treatment regimen and to address poor adherence when it occurs. These measures should be tailored to the individual patient's circumstances, based on a detailed anamnesis of the patient's clinical and social history, and be mutually acceptable to the patient and the provider. Such measures may include direct observation of medication ingestion (directly observed treatment) and identification and training of a treatment supporter (for TB and, if appropriate, for HIV-infection) who is acceptable and accountable to the patient and to the health system. Appropriate incentives and enablers, including financial, social and psychosocial supports, may also serve to enhance treatment adherence $[4,61,62]$.

\section{Standard 10 (ISTC 10 valid with an EU-adapted supplement)}

Response to therapy in patients with pulmonary TB should be monitored by follow-up smear microscopy and culture at the time of completion of the initial phase of treatment (two months for drug-susceptible TB). If the sputum smear and culture are positive at completion of the initial phase, sputum smears should be examined again at three months and, if positive, drug susceptibility testing should be performed. In patients with extrapulmonary $\mathrm{TB}$ and in children unable to produce sputum, the response to treatment is assessed clinically.

\section{EU-adapted supplement}

Treatment monitoring of MDR-TB cases should be performed based on sputum smear and culture on a monthly basis [34]. The practices for treatment monitoring should be performed according to international guidelines [40]. 
Standard 11 (replaces ISTC 11 ${ }^{\S}$, see also ESTC 1, 2 and 3)

An assessment of the likelihood of drug resistance, based on history of prior treatment, exposure to a possible source case having drug-resistant organisms and the community prevalence of drug resistance, should be obtained for all patients. Rapid testing, including rapid rifampicin and isoniazid resistance testing should be performed for all patients suspected of resistance as defined in standards 2 and 8. Furthermore, patient counselling and education should begin immediately for all TB patients, in order to minimise the potential for transmission. Infection control measures appropriate to the setting should be applied as recommended in ESTC public health standard 20.

\section{Notes}

1) This standard emphasises the need to use the WHOrecommended rapid molecular assays to rapidly rule out or confirm suspicions of MDR-TB, as described in standards 2 and 8 .

2) As expressed in standard 2, rapid molecular testing for rifampicin and isoniazid resistance does not rule out the requirement to perform standard DST to confirm results from the molecular test as well as perform the comprehensive standard DST.

\section{Standard 12 (replaces ISTC 12 $^{f}$ )}

Patients with, or highly likely to have, TB caused by drugresistant (especially MDR/extensively drug-resistant (XDR)TB) organisms should be treated with specialised regimens containing second-line anti-TB drugs. The regimen chosen may be standardised or based on suspected or confirmed drug susceptibility patterns. At least four drugs to which the organisms are known, or presumed, to be susceptible to, including an injectable agent and pyrazinamide, should be used. Treatment should be given for at least 20 months, the recommended intensive phase of treatment being 8 months (instead of 6 months as in previous recommendations; see below the EU adaptations for further detail).

\section{EU-adapted supplement}

As the treatment of $\mathrm{MDR} / \mathrm{XDR}-\mathrm{TB}$ often represents a last chance to ensure patient cure and survival, a full range of patient-centred measures, including counselling, observation and support of treatment, as well as psychosocial support are required to ensure adherence. This is particularly important given that these patients often belong to socially and economically disadvantaged groups.

For the treatment of MDR-TB, the addition of pyrazinamide to a minimum of four second-line anti-TB drugs likely to be "effective" is recommended, including the use of second-line injectable drugs, as well as the use of fluoroquinolones (earlier generations of fluoroquinolones, ciprofloxacin and ofloxacin, are no longer recommended when later generation fluoroquinolones, levofloxacin or moxifloxacin, are available) and ethionamide (or prothionamide) and either cycloserine or $\mathrm{p}$ aminosalicylic acid if cycloserine cannot be used [34].

Second-line DST should be performed to confirm the drug resistance pattern and to guide the correct choice of treatment.
Adverse events following prescription of second-line drugs should be managed according to international recommendations with the aim to limit the probability of losing an effective drug due to such adverse events [1-32].

Adverse events and the decision to start, modify or interrupt a second-line regimen should ideally be managed by a team of experts (e.g. "Consilium" or similar body) and not by individual physicians, in order to minimise mistakes and share responsibilities as well as share experience and expertise. All efforts should be made to avoid development of additional drug resistance $[4,36,37,63]$.

In addition to chemotherapy, surgery has proven to be effective in selected cases [37].

Notes

1) In order to prevent the selection of resistant $M$. tuberculosis mutants it is essential to treat with more than one effective drug and a minimum of four effective drugs. It is therefore essential not to add only one or two effective drugs to a failing regimen [36, 64-75].

\section{Standard 13 (ISTC 13 valid with an EU-adapted supplement)}

A written record of all medications given, bacteriological response and adverse reactions should be maintained for all patients.

\section{EU-adapted supplement}

At the first contact with each patient, the complete clinical and social history on TB should be collected and included in the medical records. It should include the available information on previous diagnosis, treatment (regimen, doses, duration and changes in the regimen, etc.) and compliance, as well as complete information on bacteriology at diagnosis and during follow-up (sputum smear, culture and species identification, drug susceptibility testing for first- and second-line drugs). This information should be reported in the documentation released to the patient (discharge letter, transfer-out form or equivalent document) to facilitate continuum of care if the patient is moved to another health unit $[76,77]$.

Notes

1) Reporting forms for the described documentation can be obtained from several sources, some of which are listed here [2, $12,41,78]$.

\section{STANDARDS FOR ADDRESSING HIV INFECTION AND COMORBID CONDITIONS \\ Standard 14 (replaces ISTC $14^{\star *}$ )}

HIV testing and counselling should be recommended to all patients with, or suspected of having, TB. Testing is of special importance as part of the routine management of all patients in areas with a high prevalence of HIV infection in the general population and in patients with symptoms and/or signs of HIV-related conditions. Because of the close relationship between TB and HIV infection, integrated approaches to prevention and treatment of both infections are recommended.

Notes

1) The aspect related to the setting and choice of offering HIV testing was removed from the ESTC as it was deemed not 
relevant to the EU setting. Rather, in the EU it is considered highly feasible to offer HIV testing to all TB patients. Furthermore, it is considered important that integrated TBHIV management approaches be formed in the EU setting, regardless of the prevalence [79-86].

\section{Standard 15 (replaces ISTC 15 ${ }^{\# \#)}$}

All patients with TB and HIV infection should be evaluated to determine if antiretroviral therapy is indicated during the course of treatment for TB, according to the severity of their immunodeficiency. Appropriate arrangements for access to antiretroviral drugs should be made for patients who meet indications for treatment. However, initiation of treatment for TB should not be delayed and the antiretroviral treatment prescribed as soon as possible based on evidence.

Notes

1) The consideration of treatment with cotrimoxazole was retracted from the ESTC as they refer to HIV management and prevention of other infections. General prophylactic treatment against other infections is not relevant in the EU setting. Rather, the risk of HIV-related infections must be considered individually for each patient based on risk factors and setting, and form the basis for decisions to provide prophylactic treatment against infections other than TB.

\section{Standard 16 (ISTC 16 valid with an EU-adapted supplement)}

Persons with HIV infection who, after careful evaluation, have a positive test for presumed latent infection with $M$. tuberculosis (LTBI) (TST and/or IGRAs) but do not have active TB should be treated with isoniazid for 6-9 months or any new regimen for which evidence becomes available.

\section{EU-adapted supplement}

In persons with known HIV infection in whom LTBI is confirmed or highly probable, and in whom active TB is excluded, LTBI preventive treatment with isoniazid should be provided [84, 87-90].

As HIV infection is known to increase the probability of developing TB disease upon infection, HIV-seropositive persons who have been in contact of an index case harbouring an MDR-TB strain, should undergo strict regular clinical follow-up, allowing rapid initiation of specialised treatment in case of disease development. While waiting for, or in the absence of isolation of the responsible strain, the treatment should be based on the index case's drug-susceptibility pattern (see also ESTC standards for diagnosis and treatment).

Strict clinical monitoring and no treatment of latent infection should be provided if the index case is affected by an MDR-TB strain [4].

The ESTC above should also be applicable for persons with comorbidities or on treatments that increase the risk for TB reactivation.

Notes

1) Comorbidities for reactivation of $\mathrm{TB}$, other than HIV infection, encompass conditions that decrease the host immune response, some of which are: diabetes mellitus, treatment with tumour necrosis factor (TNF)-antagonists, cancer chemotherapy and treatment with high-dose corticosteroids, etc. [90, 91]
Standard 17 (ISTC 17 valid with an EU-adapted supplement) All providers should conduct a thorough assessment of conditions that could affect TB treatment response or outcome. At the time the case management plan is developed, the provider should identify additional services that would support an optimal outcome for each patient and incorporate these services into an individualised plan of care. This plan should include assessment of, and referrals for treatment, of other illnesses with particular attention to those known to affect treatment outcome, for instance care for diabetes mellitus, drug and alcohol treatment programmes, tobacco smoking cessation programmes and other psychosocial support services, or to such services as antenatal or well-baby care.

\section{EU-adapted supplement}

Implementation of the entire package described in the WHO interim policy on collaborative TB/HIV activities should be performed for all the activities, both those covered here and in the remaining WHO package [79, 80, 82-84].

\section{STANDARDS FOR PUBLIC HEALTH AND TB PREVENTION}

\section{Standard 18 (ISTC 18 valid with an EU-adapted supplement)}

All providers of care for patients with TB should ensure that persons who are in close contact with patients who have infectious TB (e.g. in families, congregate settings like migrants shelters, schools and prisons), are evaluated and managed in line with international recommendations. The risk of $\mathrm{TB}$ transmission depends on the concentration of the mycobacteria in the air, the duration of the contact and the susceptibility of the contact to infection and disease. The determination of priorities for contact investigation is based on the likelihood that a contact: 1) has undiagnosed TB; 2) is at high risk of having been infected by the index case; 3 ) is at high risk of developing TB if infected; and 4) is at risk of having severe TB if the disease develops.

\section{EU-adapted supplement}

Individuals undergoing treatment with TNF-antagonists should be considered as high-risk contacts. According to ESTC number 16, in individuals who are HIV infected or affected by comorbidities, treatment of latent infection should be promptly initiated if TB infection is identified by TST and/ or IGRAs and active TB disease is excluded [92-96]. In case of contact with an MDR-TB index case, strict clinical monitoring should be provided, as there is no evidence that treatment of latent infection with available drugs is presently effective.

Notes

1) The bullets listing the levels of priority for initiating contact investigation as described in the ISTC have been removed from the ESTC, as they were considered not to be relevant for the EU setting. Rather, an introductory sentence was added stating the determinants of TB transmission that should be considered when assessing whether transmission has occurred and the need for initiating contact tracing $[46,60,94,95,97]$.

\section{Standard 19 (ISTC 19 valid with an EU-adapted supplement)}

Children under 5 yrs of age and persons of any age with HIV infection who are close contacts of an infectious index patient 
and who, after careful evaluation, do not have active TB, should be treated for presumed latent $\mathrm{TB}$ infection with isoniazid.

\section{EU-adapted supplement}

As per previous ESTC, strict clinical monitoring and no treatment of latent infection should be provided if the index case is affected by an MDR-TB strain [4].

Clinicians should collaborate with public health authorities in implementing adequate contact tracing procedures, performed according to national and international recommendations on progressive circles, when an infectious index case is diagnosed and notified [94, 98-100].

\section{Notes}

1) Preventive treatment for presumed latent $T B$ infection should be according to national and international recommendations [87, 89].

\section{Standard 20 (ISTC 20 valid with an EU-adapted supplement)}

Each healthcare facility caring for patients who have, or are suspected of having infectious TB, should develop and implement an appropriate TB infection control plan.

\section{EU-adapted supplement}

Clinicians should ensure that all newly admitted patients who are suspected of having infectious TB are subject to respiratory isolation until their diagnosis is confirmed and excluded [4].

In order to prevent transmission of mycobacteria to other patients, staff and/or visitors and smear-positive TB patients should ideally be isolated in appropriate rooms until they achieve bacteriological conversion (negative-sputum microscopy). Patients suspected of having TB (if feasible) and MDRTB patients (strongly recommended) should be isolated in negative-pressure ventilation rooms.

An appropriate infection control plan, managed by a designated person, should include the following four components: managerial activities, administrative controls, environmental controls and personal protection interventions [97]. Adequate administrative measures for TB infection control should be in place in all healthcare facilities, as well as adequate respiratory protection measures (including the use of respirators following respirator fit testing for staff and the use of surgical mask for infectious patients). Appropriate training on infection control to staff, and standardised health education of patients on cough etiquette, based on validated tools, should also be included in the infection control plan. Infection control committees, which cover airborne diseases, and includes infection control experts, should also be implemented [60, 97].

\section{Notes}

1) The implementation of an infection control plan is essential for the treating clinician, health facility and the overall health system. Clinicians should maintain dialogue within their health facility; develop a sound infection control plan, and their technical expertise. The health facility should engage with all healthcare workers, non-medical staff, patients and visitors and ensure optimal implementation, practice and monitoring of these infection control measures; all healthcare workers should be (re)trained in the infection control plan $[4,38,97$, 101].

2) With regard to the need of isolating infectious TB patients, it is important to consider several options for isolation, and not only that of hospitalisation. For example, a patient with drugsusceptible TB that can be treated at home (i.e. no need for hospitalisation due to severe health status), does not need to be hospitalised, as long as appropriate measures for treatment and infection control are ensured at the residence [97, 102, 103].

3) Patients with a clinical indication for hospital admission, such as comorbidities, should not be hospitalised in a general medical ward. Ideally such patients should be placed in rooms that allow appropriate respiratory isolation. It is therefore important to have a designated infection control focal person with the required authority to ensure implementation of the infection control plan.

Standard 21 (ISTC 21 valid with an EU-adapted supplement) All providers must report both new and retreatment TB cases and their treatment outcomes to local public health authorities, in conformance with applicable legal requirements and policies.

\section{EU-adapted supplement}

Clinicians should perform treatment outcome evaluations in their clinical unit at regular time intervals (e.g. quarterly) [6, 104, 105]. Treatment outcomes should be reported to local public health authorities, in conformance with applicable requirements and policies and, at the same time be used as a monitoring and evaluation tool to improve the quality of patient management. Information on treatment outcome should also regularly be channelled back from the public health department to the healthcare providers, to allow a coordinated evaluation of the outcomes. Information on the final outcome of patients should be available at the clinical unit which initiated treatment, even when the patient is transferred out. Adequate training must be provided to health staff in charge of reporting treatment outcomes to public health authorities and performing the quarterly evaluation of own cases. This principle is also applicable to TB patients moving across the EU borders [5, 13, 25, 106, 107].

\section{SUPPORTING ENABLERS TO THE ESTC}

In the process of developing the standards and reaching a consensus, the panel of experts identified the need and added value of identifying and listing supporting enablers to the standards. These are a resource for policy makers, clinicians, public health workers and other stakeholders to identify how best to adopt, adapt, introduce and implement the ESTC in their setting with the ultimate goal of securing optimal TB care, prevention and control $[2,4,12,38,60]$. They are as follows:

1) Formal adoption of the European Standards for Tuberculosis Care, for the care, prevention and control, ideally translated into the country-specific language(s) after their endorsement by national medical associations. Ideally the ESTC should be incorporated into training curricula of health staff [108].

2) Development of consistent $\mathrm{TB}$ control and elimination strategies and policies according to the principles described in 
the new European Framework [7] and the Framework action plan to fight TB in the EU and its follow-up [109-112].

3) Adoption of specific, updated, evidence-based TB and MDR-TB guidelines, together with mechanisms to update them on a regular basis and to monitor their implementation (audit- and or knowledge, attitudes and practices study-based) $[63,113,114]$.

4) Planning and organisation of an adequate national laboratory network to ensure that a minimum and sufficient number of mycobacteriology laboratories are in place, allowing implementation of the standards described in this document (adequate coverage of the country, adequate internal and external quality assurance procedures in place, sufficient numbers of samples per laboratory to ensure proficiency, availability of national laboratories with reference functions to support regional and local laboratories etc.).

5) Development of policies ensuring a continuous availability of all first- and second-line TB drugs (e.g. through coordinated procurement with partner countries for the drugs not registered in the country or which are necessary in small quantities) $[19,110,115]$.

6) Securing consistent and adequate funding for TB and MDR/ $\mathrm{XDR}-\mathrm{TB}$ care, prevention and control that is sufficient to run the activities mentioned in this document. This should include psychosocial support and coordination of care for all patients, as highlighted in the International Patients' Charter for rights to diagnosis and treatment. This applies particularly to patients belonging to vulnerable populations [57, 107, 116-118].

\section{REPLACED ISTC}

"ISTC 1: all persons with otherwise unexplained productive cough lasting 2-3 weeks or more should be evaluated for TB.

\#ISTC 2: all patients (adults, adolescents and children who are capable of producing sputum) suspected of having pulmonary TB should have at least two sputum specimens submitted for microscopic examination in a quality-assured laboratory. When possible, at least one early morning specimen should be obtained.

IISTC 3: for all patients (adults, adolescents and children) suspected of having extrapulmonary TB, appropriate specimens from the suspected sites of involvement should be obtained for microscopy, culture and histopathological examination.

${ }^{+}$ISTC 4: all persons with chest radiographic findings suggestive of TB should have sputum specimens submitted for microbiological examination.

${ }^{\S}$ ISTC 11: an assessment of the likelihood of drug resistance, based on history of prior treatment, exposure to a possible source case having drug-resistant organisms and the community prevalence of drug resistance, should be obtained for all patients. DST should be performed at the start of therapy for all previously treated patients. Patients who remain sputum smear-positive at completion of three months of treatment and patients who have failed, defaulted from, or relapsed following one or more courses of treatment should always be assessed for drug resistance. For patients in whom drug resistance is considered to be likely, culture and testing for susceptibility / resistance to at least isoniazid and rifampicin should be performed promptly. Patient counselling and education should begin immediately to minimise the potential for transmission. Infection control measures appropriate to the setting should be applied.

${ }^{f}$ ISTC 12: patients with, or highly likely to have, TB caused by drug-resistant (especially MDR/XDR) organisms should be treated with specialised regimens containing second-line antiTB drugs. The regimen chosen may be standardised or based on suspected or confirmed drug susceptibility patterns. At least four drugs to which the organisms are known or presumed to be susceptible, including an injectable agent, should be used and treatment should be given for at least 1824 months beyond culture conversion. Patient-centred measures, including observation of treatment, are required to ensure adherence. Consultation with a provider experienced in treatment of patients with MDR/XDR-TB should be obtained.

${ }^{* *}$ ISTC 14: HIV testing and counselling should be recommended to all patients with, or suspected of having, TB. Testing is of special importance as part of routine management of all patients in areas with a high prevalence of HIV infection in the general population, in patients with symptoms and/or signs of HIV-related conditions, and in patients having a history suggestive of a high risk of HIV exposure. Because of the close relationship between TB and HIV infection, in areas of high HIV prevalence integrated approaches to prevention and treatment of both infections are recommended.

\#\#ISTC 15: all patients with TB and HIV infection should be evaluated to determine if antiretroviral therapy is indicated during the course of treatment for TB. Appropriate arrangements for access to antiretroviral drugs should be made for patients who meet indications for treatment. However, initiation of treatment for TB should not be delayed. Patients with TB and HIV infection should also receive cotrimoxazole as prophylaxis for other infections.

\section{SUPPORT STATEMENT}

The development of the ESTC was financially supported by the European Centre for Disease Prevention and Control (ECDC) service contract with the European Respiratory Society (ERS) (reference: ECD2691).

\section{STATEMENT OF INTEREST}

Statements of interest for Z.P. Zellweger, D.M. Cirillo, and V.Ø. Thomsen can be found at www.erj.ersjournals.com/site/misc/ statements.xhtml

\section{ACKNOWLEDGEMENTS}

The authors would like to thank A. Turnbull and C. Pannetier (ERS Office, Lausanne, Switzerland) for making this project possible.

The affiliations for the Task Force members are as follows: G.B. Migliori, L. D'Ambrosio and R. Centis: WHO Collaborating Centre for TB and Lung Diseases, Fondazione S. Maugeri, Care and Research Institute, Tradate, Italy. J.P. Zellweger: TB Competence Centre, Swiss Lung Association, Bern, Switzerland. I. Abubakar: Tuberculosis Section, Health Protection Services, Health Protection Agency, London, and Norwich Medical School, Norwich, UK. E. Ibraim: National Tuberculosis Programme, Marius Nasta Institute of Pulmonology, Bucharest, Romania. J.A. Caminero: Dept of Pulmonary Medicine, Hospital General de Gran Canaria "Dr. Negrın", Las Palmas 
de Gran Canaria, Spain and International Union against Tuberculosis and Lung Disease, Paris, France. G. De Vries: Country Office The Netherlands, KNCV Tuberculosis Foundation, The Hague, and Centre for Infectious Disease Control, National Institute for Public Health and the Environment, Bilthoven, the Netherlands. G. Sotgiu: Epidemiology and Medical statistics Unit, Dept of Biomedical Sciences, University of Sassari, Sassari, Italy. O. Menegale: ERS Headquarters, Lausanne, Switzerland. K. Kliiman: Dept of Pulmonary Medicine, University of Tartu, Tartu, Estonia. T. Aksamit: Pulmonary Disease and Critical Care Medicine, Mayo Clinic Rochester, Rochester, MN, USA. D.M. Cirillo: Emerging Bacterial Pathogens Unit, San Raffaele Scientific Institute, Milan, Italy. M. Danilovits: Dept of Tuberculosis, Tartu University Hospital, Lung, Estonia. M. Dara and H. Kluge: Division of Communicable Diseases, Health Security and Environment, World Health Organization, Regional Office for Europe, Copenhagen, Denmark. K. Dheda: Lung Infection and Immunity Unit, Division of Pulmonology and University of Cape Town Lung Institute, Dept of Medicine, Cape Town, South Africa. A.T. Dinh-Xuan: Dept of Cardiopulmonary Medicine, Cochin Hospital, University Paris Descartes, Paris, France. C. Lange: Division of Clinical Infectious Diseases, Medical Clinic, Research Centre Borstel, and Tuberculosis Network European Trial group (TBNET), Borstel, Germany. V. Leimane: State Agency Infectology Centre, Tuberculosis and Lung Disease Clinic Riga, Riga, Latvia. R. Loddenkemper: German Central Committee against Tuberculosis (DZK), Berlin, Germany. L.P. Nicod: Faculté de Biologie et de Médecine, et de Pneumologie, Centre Hospitalier Universitaire Vaudois, Lausanne, Switzerland. M.C. Raviglione: World Health Organization, Geneva, Switzerland. A. Spanevello: Fondazione S. Maugeri, Care and Research Institute, Tradate, University of Insubria, Varese, Italy. V.Ø. Thomsen: International Reference Laboratory of Mycobacteriology, Statens Serum Institut, Copenhagen, Denmark. M. Villar: General Directorate of Health in Lisbon and Lung Diseases Centre of Venda Nova, Amadora, Portugal. M. Wanlin: Belgian Lung and Tuberculosis Association, Brussels, Belgium. J.A. Wedzicha: UCL Medical School, University College London, London, UK. A. Zumla: Division of Infection and Immunity, Dept of Infection, University College London Medical School, London, UK. F. Blasi: Respiratory Medicine Section, Dipartimento Toraco-Polmonare e Cardiocircolatorio, University of Milan, IRCCS Fondazione Cà Granda, Milan, Italy. E. Huitric, A. Sandgren and D. Manissero: European Centre for Disease Prevention and Control, Stockholm, Sweden.

\section{REFERENCES}

1 Migliori GB, Sotgiu G, Blasi F, et al. Towards the development of EU/EEA Standards for Tuberculosis Care (ESTC). Eur Respir J 2011; 38: 493-495.

2 Migliori GB, Sotgiu G, D'Ambrosio L, et al. TB and MDR/XDRTB in the EU and EEA countries: managed or mismanaged? Eur Respir J 2012; [Epub ahead of print DOI: 10.1183/ 09031936.00170411].

3 Abubakar I, Dara M, Manissero D. Tackling the spread of drugresistant tuberculosis in Europe. Lancet 2012; 379: e21-e23.

4 Tuberculosis Coalition for Technical Assistance. International Standards for Tuberculosis Care (ISTC), 2nd Edn. The Hague, Tuberculosis Coalition for Technical Assistance, 2009.

5 Rieder HL, Watson JM, Raviglione MC, et al. Surveillance of tuberculosis in Europe. Working Group of the World Health Organization (WHO) and the European Region of the International Union Against Tuberculosis and Lung Disease (IUATLD) for uniform reporting on tuberculosis cases. Eur Respir J 1996; 9: 1097-1104.

6 Veen J, Raviglione M, Rieder HL, et al. Standardized tuberculosis treatment outcome monitoring in Europe. Recommendations of a Working Group of the World Health Organization (WHO) and the European Region of the International Union Against Tuberculosis and Lung Disease (IUATLD) for uniform reporting by cohort analysis of treatment outcome in tuberculosis patients. Eur Respir J 1998; 12: 505-510.

7 Broekmans JF, Migliori GB, Rieder HL, et al. European framework for tuberculosis control and elimination in countries with a low incidence. Recommendations of the World Health Organization (WHO), International Union Against Tuberculosis and Lung Disease (IUATLD) and Royal Netherlands Tuberculosis Association (KNCV) Working Group. Eur Respir J 2002; 19: 765-775.

8 Clancy L, Rieder HL, Enarson DA, et al. Tuberculosis elimination in the countries of Europe and other industrialized countries. Eur Respir J 1991; 4: 1288-1295.

9 Schwoebel V, Lambregts-van Weezenbeek CS, Moro ML, et al. Standardization of antituberculosis drug resistance surveillance in Europe. Recommendations of a World Health Organization (WHO) and International Union Against Tuberculosis and Lung Disease (IUATLD) Working Group. Eur Respir J 2000; 16: 364-371.

10 Drobniewski FA, Hoffner S, Rusch-Gerdes $S$, et al. Recommended standards for modern tuberculosis laboratory services in Europe. Eur Respir J 2006; 28: 903-909.

11 Veen J, Migliori GB, Raviglione M, et al. Harmonisation of TB control in the WHO European region: the history of the Wolfheze Workshops. Eur Respir J 2011; 37: 950-959.

12 Facchini A, D'Ambrosio L, Sotgiu G, et al. Is management of MDR-/XDR-TB in Europe adequate? A TBNET survey. Eur Respir J 2010; 36: Suppl. 52, 32s.

13 European Centre for Disease Prevention and Control. Framework Action Plan to fight tuberculosis in the European Union. Stockholm, ECDC, 2008.

14 European Centre for Disease Prevention and Control. Progressing towards TB elimination. Stockholm, ECDC, 2010.

15 Storla DG, Yimer S, Bjune GA. A systematic review of delay in the diagnosis and treatment of tuberculosis. BMC Public Health 2008; 8: 15 .

16 Loddenkemper R, Gibson GJ, Sibille Y, eds. European Respiratory Society/European Lung Foundation. Lung Health in Europe Facts and Figures. Sheffield, ERSJ, 2003.

17 Schwoebel V, Lambraegts-van Weezenbeeck CSB, Moro ML, et al. European recommendations on surveillance of antituberculosis drug resistance. Euro Surveill 2000; 5: 104-106.

18 World Health Organization. Multidrug and extensively drugresistant TB (M/XDR-TB). 2010 Global report on surveillance and response. WHO/HTM/TB/2010.3. Geneva, Switzerland, 2010.

19 World Health Organization. Report of the Tenth Meeting WHO Strategic and Technical Advisory Group for Tuberculosis (STAG-TB) 27-29 September 2010. WHO/HTM/TB/2010.18. Geneva, World Health Organization, 2010.

20 Boehme CC, Nabeta P, Hillemann D, et al. Rapid molecular detection of tuberculosis and rifampin resistance. $N$ Engl J Med 2010; 363: 1005-1015.

21 Boehme CC, Nicol MP, Nabeta P, et al. Feasibility, diagnostic accuracy, and effectiveness of decentralised use of the Xpert MTB/RIF test for diagnosis of tuberculosis and multidrug resistance: a multicentre implementation study. Lancet 2011; 377: 1495-1505.

22 Klinkenberg E, Manissero D, Semenza JC, et al. Migrant tuberculosis screening in the EU/EEA: yield, coverage and limitations. Eur Respir J 2009; 34: 1180-1189.

23 Arshad S, Bavan L, Gajari K, et al. Active screening at entry for tuberculosis among new immigrants: a systematic review and meta-analysis. Eur Respir J 2010; 35: 1336-1345.

24 Caminero JA. Multidrug-resistant tuberculosis: epidemiology, risk factors and case finding. Int J Tuberc Lung Dis 2010; 14: 382-390. 
25 ECDC and WHO Regional Office for Europe. Surveillance Report. Tuberculosis Surveillance in Europe 2008. Stockholm, European Centre for Disease Prevention and Control, 2010.

26 Eker B, Ortmann J, Migliori GB, et al. Multi-drug resistant and extensively drug resistant tuberculosis, Germany. Emerg Infect Dis 2008; 14: 1700-1706.

27 Migliori GB, Besozzi G, Girardi E, et al. Clinical and operational value of the extensively drug-resistant tuberculosis definition. Eur Respir J 2007; 30: 623-626.

28 Sotgiu G, Ferrara G, Matteelli A, et al. Epidemiology and clinical management of XDR-TB: a systematic review by TBNET. Eur Respir J 2009; 33: 871-881.

29 World Health Organization. Report of the Eight Meeting WHO Strategic and Technical Advisory Group for Tuberculosis (STAG-TB) 23-25 June 2008. Geneva, World Health Organization, 2008. Available at www.who.int/tb/events/stag report_2008.pdf. Date last accessed: June 30, 2011.

30 Ling DI, Zwerling AA, Pai M. GenoType MTBDR assays for diagnosis of multidrug-resistant tuberculosis: a meta-analysis. Eur Resp J 2008; 32: 1165-1174.

31 World Health Organization. Report of the Eleventh Meeting WHO Strategic and Technical Advisory Group for Tuberculosis (STAG-TB) 20-22 June 2011. Geneva, World Health Organization, 2011. In press.

32 Migliori GB, Raviglione MC, Schaberg T, et al. Tuberculosis management in Europe. Task Force of the European Respiratory Society (ERS), the World Health Organisation (WHO) and the International Union against Tuberculosis and Lung Disease (IUATLD) Europe Region. Eur Respir J 1999; 14: 978-992.

33 World Health Organization. Guidelines for the programmatic management of drug-resistant tuberculosis. WHO/HTM/TB/ 2008.402. Geneva, World Health Organization, 2008.

34 Falzon D, Jaramillo E, Schünemann HJ, et al. WHO guidelines for the programmatic management of drug-resistant tuberculosis: 2011 update. Eur Respir J 2011; 38: 516-528.

35 National Institute of Clinical Excellence (NICE). Tuberculosis: clinical diagnosis and management of tuberculosis, and measures of its prevention and control. London, NICE, 2011.

36 Caminero JA. Treatment of multidrug-resistant tuberculosis: evidence and controversies. Int J Tuberc Lung Dis 2006; 10: 829837.

37 Caminero JA, Sotgiu G, Zumla A, et al. Best drug treatment for multidrug-resistant and extensively drug-resistant tuberculosis. Lancet Infect Dis 2010; 10: 621-629.

38 Sotgiu G, Centis R, D'Ambrosio L, et al. Development of a standardised tool to survey MDR-/XDR-TB case management in Europe. Eur Respir J 2010; 36: 208-211.

39 World Health Organization. Toman's tuberculosis: case detection, treatment, and monitoring. 2nd Edn. WHO/HTM/TB/ 2004.334. Geneva, World Health Organization, 2004.

40 World Health Organization. Treatment of tuberculosis: guidelines. 4th Edn. WHO/HTM/TB/2009.420. Geneva, World Health Organization, 2009.

41 European Union Commission. 2008/426/EC: commission decision of 28 April 2008 amending decision 2002/253/EC laying down case definitions for reporting communicable diseases to the Community network under decision No 2119/98/EC of the European Parliament and of the Council (notified under document number C(2008) 1589).OJ L 159, 18.06.2008., p. 46. Comissions EU definition). Off J Eur Union 2008; 46.

42 Schoch OD, Rieder P, Tueller C, et al. Diagnostic yield of sputum, induced sputum, and bronchoscopy after radiologic tuberculosis screening. Am J Respir Crit Care Med 2007; 175: 80-86.

43 Jafari C, Kessler P, Sotgiu G, et al. Impact of a Mycobacterium tuberculosis-specific interferon- $\gamma$ release assay in bronchoalveolar lavage fluid for a rapid diagnosis of tuberculosis. J Intern Med 2011; 270: 254-262.
44 Lange C, Mori T. Advances in the diagnosis of tuberculosis Respirology 2010; 15: 220-240.

45 American Thoracic Society/Centers for Disease Control and Prevention/Infectious Diseases Society of America. Treatment of tuberculosis. Am J Respir Crit Care Med 2003; 167: 603-662.

46 European Centre for Disease Prevention and Control. Use of interferon-gamma release assays in support of TB diagnosis. Stockholm, ECDC, 2011.

47 World Health Organization. WHO Policy statement: molecular line probe assays for rapid screening of patients at risk of multidrug-resistant tuberculosis (MDR-TB). Geneva, World Health Organization, 2008

48 Diel R, Goletti D, Ferrara G, et al. Interferon- $\gamma$ release assays for the diagnosis of latent Mycobacterium tuberculosis infection: a systematic review and meta-analysis. Eur Respir J 2011; 37: 88-99.

49 Sester M, Sotgiu G, Lange C, et al. Interferon- $\gamma$ release assays for the diagnosis of active tuberculosis: a systematic review and meta-analysis. Eur Respir J 2011; 37: 100-111.

50 Pai M, Flores LL, Pai N, et al. Diagnostic accuracy of nucleic acid amplification tests for tuberculous meningitis: a systematic review and meta-analysis. Lancet Infect Dis 2003; 3: 633-643.

51 Pai M, Ramsay A, O'Brien R. Evidence-based tuberculosis diagnosis. PLoS Med 2008; 5: e156.

52 Martin A, Portaels F, Palomino JC. Colorimetric redox-indicator methods for the rapid detection of multidrug resistance in Mycobacterium tuberculosis: a systematic review and metaanalysis. J Antimicrob Chemother 2007; 59: 175-183.

53 Ling DI, Flores LL, Riley LW, et al. Commercial nucleic-acid amplification tests for diagnosis of pulmonary tuberculosis in respiratory specimens: meta-analysis and meta-regression. PLOS ONE 2008; 3: e1536.

54 Kik SV, Franken WPJ, Mensen M, et al. Predictive value for progression to tuberculosis by IGRA and TST in immigrant contacts. Eur Respir J 2010; 35: 1346-1353.

55 World Health Organization. Rapid advice. Treatment of tuberculosis in children. WHO/HTM/TB/2010.13:1-19. Geneva, World Health Organization, 2010.

56 World Health Organization. Report of a WHO consultation on strengthening the active engagement of civil society organizations in the global TB prevention, care and control efforts. WHO/ HTM/TB/2010.15:1-7. Geneva, World Health Organization, 2010

57 Patients' Charter for Tuberculosis Care. World Care Council (C)2010. Available at www.worldcarecouncil.org Date last accessed: June 30, 2011.

58 Yew WW, Lange C, Leung CC. Treatment of tuberculosis: update 2010. Eur Respir J 2011; 37: 441-462.

59 Oxlade O, Schwartzman K, Pai M, et al. Predicting outcomes and drug resistance with standardised treatment of active tuberculosis. Eur Respir J 2010; 36: 870-877.

60 Sotgiu G, D'Ambrosio L, Centis R, et al. TB and M/XDR-TB infection control in European TB reference centres: the Achilles' heel? Eur Respir J 2011; 38: 1221-1223.

61 Munro SA, Lewin SA, Smith HJ, et al. Patient adherence to tuberculosis treatment: a systematic review of qualitative research. PLoS Med 2007; 4: e238.

62 Volmink J, Matchaba P, Garner P. Directly observed therapy and treatment adherence. Lancet 2000; 355: 1345-1350.

63 World Health Organization. Implementing the Stop TB strategy: A handbook for national tuberculosis control programmes. WHO/HTM/TB/2008.401. Geneva, WHO, 2008.

64 Espinal MA, Kim SJ, Suarez PG, et al. Standard short-course chemotherapy for drug-resistant tuberculosis: treatment outcomes in 6 countries. JAMA 2000; 283: 2537-2545.

65 Migliori GB, Espinal MA, Danilova ID, et al. Frequency of recurrence among MDR-TB cases "successfully" treated with 
standardised short-course chemotherapy. Int J Tuberc Lung Dis 2002; 6: 858-864.

66 Gillespie SH. Evolution of drug resistance in Mycobacterium tuberculosis: clinical and molecular perspective. Antimicrob Agents Chemother 2002; 46: 267-274.

67 Leimane V, Dravniece G, Riekstina V, et al. Treatment outcome of multidrug/extensively drug-resistant tuberculosis in Latvia, 2000-2004. Eur Respir J 2010; 36: 584-593.

68 Kim DH, Kim HJ, Park SK, et al. Treatment outcomes and survival based on drug resistance patterns in multidrug-resistant tuberculosis. Am J Respir Crit Care Med 2010; 182: 113-119.

69 Lew W, Pai M, Oxlade O, et al. Initial drug resistance and tuberculosis treatment outcomes: systematic review and metaanalysis. Ann Intern Med 2008; 149: 123-134.

70 Caminero JA. Likelihood of generating MDR-TB and XDR-TB under adequate national tuberculosis control programme implementation. Int J Tuberc Lung Dis 2008; 12: 869-877.

71 Kim HR, Hwang SS, Kim HJ, et al. Impact of extensive drug resistance on treatment outcomes in non-HIV-infected patients with multidrug-resistant tuberculosis. Clin Infect Dis 2007; 45: 1290-1295.

72 Migliori GB, Sotgiu G, Lange C, et al. Extensively drug-resistant tuberculosis: back to the future. Eur Respir J 2010; 36: 475-477.

73 Shin SS, Keshavjee S, Gelmanova IY, et al. Development of extensively drug-resistant tuberculosis during multidrug-resistant tuberculosis treatment. Am J Respir Crit Care Med 2010; 182: 426-432.

74 Villar M, Sotgiu G, D'Ambrosio L, et al. Linezolid safety, tolerability and efficacy to treat multidrug- and extensively drug-resistant tuberculosis. Eur Respir J 2011; 38: 730-733.

75 Migliori GB, Eker B, Richardson MD, et al. A retrospective TBNET assessment of linezolid safety, tolerability and efficacy in multidrug-resistant tuberculosis. Eur Respir J 2009; 34: 387-393.

76 Chiang $\mathrm{CY}$, Bai KJ, Lee CN, et al. Inconsistent dosing of antituberculosis drugs in Taipei, Taiwan. Int J Tuberc Lung Dis 2010; 14: 878-883.

77 Caminero JA. Management of multidrug-resistant tuberculosis and patients in retreatment. Eur Respir J 2005; 25: 928-936.

78 World Health Organization. Revised TB recording and reporting forms and registers - version 2006. WHO/HTM/TB/2006.373. Geneva, World Health Organization, 2006.

79 Kruijshaar ME, Pimpin L, Abubakar I, et al. The burden of TBHIV in the EU: how much do we know? A survey of surveillance practices and results. Eur Respir J 2011; 38: 1374-1381.

80 Pimpin L, Drumright LN, Kruijshaar ME, et al. Tuberculosis and HIV co-infection in European Union and European Economic Area countries. Eur Respir J 2011; 38: 1382-1392.

81 Pontali E, Pasticci MB, Matteelli A, et al. Tuberculosis and TB/ HIV co-infection: do we have a surveillance system in Europe? Eur Respir J 2011; 38: 1258-1260.

82 World Health Organization. Interim Policy on Collaborative TB/ HIV Activities. WHO/HTM/TB2004.330-WHO/HTM/HIV/ 2004. Geneva, World Health Organization, 2004.

83 de Colombani P, Banatvala N, Zaleskis R, et al. European framework to decrease the burden of TB/HIV. Eur Respir J 2004; 24: 493-501.

84 Sester M, Giehl C, McNerney R, et al. Challenges and perspectives for improved management of HIV/Mycobacterium tuberculosis co-infection. Eur Respir J 2010; 36: 1242-1247.

85 UNAIDS/WHO. Guidance on provider-initiated HIV testing and counseling in health facilities. Geneva, World Health Organization, 2007.

86 World Health Organization. WHO 3 I's meeting: Intensified case finding (ICF), isoniazid preventive therapy (IPT), and TB infection control (IC) for people living with HIV. Geneva, World Health Organization, 2008.
87 Leung CC, Rieder HL, Lange C, et al. Treatment of latent infection with Mycobacterium tuberculosis: update 2010. Eur Respir J 2011; 37: 690-711.

88 Schutz C, Meintjes G, Almajid F, et al. Clinical management of tuberculosis and HIV-1 co-infection. Eur Respir J 2010; 36: 1460-1481.

89 Woldebanna S, Volmink J. Treatment of Latent tuberculosis infection in HIV infected persons. The Cochrane Library, 2008.

90 Solovic I, Sester M, Gomez-Reino JJ, et al. The risk of tuberculosis related to tumor necrosis factor antagonist therapies: a TBNET consensus statement. Eur Respir J 2010; 36: 1185-1206.

91 Creswell J, Raviglione MC, Ottmani S, et al. Tuberculosis and noncommunicable diseases: neglected links and missed opportunities. Eur Respir I 2011; 37: 1269-1282.

92 Lange C, Rieder HL. Intention to test is intention to treat. Am J Respir Crit Care Med 2011; 183: 3-4.

93 Bothamley GH, Ditiu L, Migliori GB, et al. Active case finding of tuberculosis in Europe: a Tuberculosis Network European Trials Group (TBNET) survey. Eur Respir J 2008; 32: 1023-1030.

94 Erkens CG, Kamphorst M, Abubakar I, et al. Tuberculosis contact investigation in low prevalence countries: a European consensus. Eur Respir J 2010; 36: 925-949.

95 Diel R, Goletti D, Ferrara G, et al. Interferon- $\gamma$ release assays for the diagnosis of latent Mycobacterium tuberculosis infection: a systematic review and meta-analysis. Eur Respir J 2011; 37: 88-99.

96 Mack U, Migliori GB, Sester M, et al. LTBI: latent tuberculosis infection or lasting immune responses to $M$. tuberculosis? A TBNET consensus statement. Eur Respir J 2009; 33: 956-973.

97 WHO policy on TB infection control in health-care facilities, congregate settings and households. WHO/HTM/TB/2009.419. Geneva, World Health Organization, 2009.

98 Horsburgh CR Jr, O'Donnell M, Chamblee S, et al. Revisiting rates of reactivation tuberculosis: a population-based approach. Am J Respir Crit Care Med 2010; 182: 420-425.

99 Newton SM, Kampmann B. Paediatric tuberculosis. Lancet Infect Dis 2008; 8: 498-510.

100 Donald PR. A research agenda to promote the management of childhood tuberculosis within national tuberculosis programmes. Int J Tuberc Lung Dis 2007; 11: 370-380.

101 Marica C, Didilescu C, Galie N, et al. Reversing the tuberculosis upwards trend: a success story in Romania. Eur Respir J 2009; 33: 168-170

102 Jensen PA, Lambert LA, Iademarco MF, et al. Guidelines for preventing the transmission of Mycobacterium tuberculosis in health-care settings, 2005. MMWR Recomm Rep 2005; 54: 1-141.

103 Nardell E, Dharmadhikari A. Turning off the spigot: reducing drug-resistant tuberculosis transmission in resource-limited settings. Int J Tuberc Lung Dis 2010; 14: 1233-1243.

104 Laserson KF, Thorpe LE, Leimane V, et al. Speaking the same language: treatment outcome definitions for multidrug-resistant tuberculosis. Int J Tuberc Lung Dis 2005; 9: 640-645.

105 World Health Organization, International Union Against Tuberculosis and Lung Disease, Royal Netherlands Tuberculosis Association. Revised international definitions in tuberculosis control. Int J Tuberc Lung Dis 2001; 5: 213-215.

106 Mor Z, Migliori GB, Althomsons SP, et al. Comparison of tuberculosis surveillance systems in low-incidence industrialised countries. Eur Respir J 2008; 32: 1616-1624.

107 Heldal E, Kuyvenhoven JV, Wares F, et al. Diagnosis and treatment of tuberculosis in undocumented migrants in low- or intermediate-incidence countries. Int J Tuberc Lung Dis 2008; 12: 878-888.

108 Williams G, Alarcon E, Jittimanee S, et al. Guidance for the implementation of best practice for the care of patients with tuberculosis. Int J Tuberc Lung Dis 2008; 12: 236-240. 
109 Nathanson E, Nunn P, Uplekar M, et al. MDR tuberculosiscritical steps for prevention and control. N Engl J Med 2010; 363 1050-1058.

110 Raviglione MC, Uplekar MW. WHO's new Stop TB Strategy. Lancet 2006; 367: 952-955.

111 Stop TB Partnership and World Health Organization. Global Plan to Stop TB 2006-2015. WHO/HTM/STB/2006.35. Geneva, World Health Organization, 2006.

112 Dye C, Watt CJ, Bleed DM, et al. The evolution of tuberculosis control, and prospects for reaching the millennium development goals. JAMA 2005; 293: 2767-2775.

113 World Health Organization. Engaging all health care providers in TB control: guidance on implementing public-private mix approaches. WHO/HTM/TB/2006.360. Geneva, World Health Organization, 2006.
114 World Health Organization. Public Private Mix for TB care and control: A tool for national situation assessment. WHO/HTM/ TB/2007.391. Geneva, World Health Organization, 2007.

115 Mitnick CD, Castro KG, Harrington M, et al. Randomized trials to optimize treatment of multidrug-resistant tuberculosis. PLoS Med 2007; 4: e292.

116 Marais BJ, Raviglione MC, Donald PR, et al. Scale-up of services and research priorities for diagnosis, management, and control of tuberculosis: a call to action. Lancet 2010; 375: 2179-2191.

117 Lönnroth K, Castro KG, Chakaya JM, et al. Tuberculosis control and elimination 2010-50: cure, care, and social development. Lancet 2010; 375: 1814-1829.

118 Migliori GB, Richardson MD, Lange C. Of blind men and elephants: making sense of extensively drug-resistant tuberculosis. Am J Respir Crit Care Med 2008; 178: 1000-1001. 